\title{
Why and How to Leverage the Commercial Space Sector for the Benefit of Planetary Science and Its Community
}

White Paper Submission for the 2023-2032 Planetary Science and Astrobiology Decadal Survey

\author{
Authors \\ Elizabeth A. Frank, First Mode \& LEAG Commercial Advisory Board Chair \\ (elizabeth@firstmode.com) \\ Casey Dreier, The Planetary Society \\ Benton Clark, Space Science Institute \\ Clive R. Neal, University of Notre Dame
}

\section{Co-signers}

Paul K. Byrne, North Carolina State University

Amy Fagan, Western Carolina University

Angeliki Kapoglou, UCL / ESA / Open Lunar

Kurt Klaus, Lunar \& Planetary Institute

Rachel Klima, Johns Hopkins APL

Peter James, Baylor University

Angela G. Marusiak, Caltech / NASA Jet Propulsion Laboratory

Paul B. Niles, NASA Johnson Space Center

Philip J. Stooke, Western University

Ryan Watkins, Planetary Science Institute

David Huntsman, NASA Glenn

Samuel Lawrence, NASA JSC

Kyle Acierno, iSpace Technologies U.S.

Billy Edwards, UCL / Blue Skies Space Ltd.

Juan Flores, Americans for Space Development

Laura Forczyk, Astralytical

Justin Kugler, Made In Space, Inc.

Melissa Lane, Fibernetics LLC

Siddharth Krishnamoorthy, Caltech /JPL

Trent Martin, Intuitive Machines

Dennis Poulos, Poulos Air \& Space, Inc. Jeff Rich, Xplore Inc.
Vickie Siegel, Stone Aerospace

Dennis Wingo, Skycorp Incorporated

Kris Zacny, Honeybee Robotics

Dave Brody, Starsong Media Inc.

Indhu Varatharajan, German Aerospace Center Arno Wieders, Space Horizon Erika Kohler, NASA Goddard Tanya Harrison, Planet Labs

Brian Y. Wu, Stanford University

Petr Brož, Inst. of Geophysics of the CAS

Brendan A. Anzures, Brown University

Don R. Weidner, Formidable Ventures Nathan Stein, Caltech

Jim Bell, Arizona State University

William C. Stone, Stone Aerospace

Marcella Yant, Lockheed Martin

Jeffrey R. Hopkins, Farsight Technologies

Julie Stopar, Lunar and Planetary Institute Rob Lillis, UC Berkeley

Jason Aspiotis, Finsophy PBC

Mark Robinson, Arizona State University

George Sowers, Colorado School of Mines

Jessica Barnes, University of Arizona 


\section{Introduction}

The past few years have been an exciting time for planetary science: NASA's Planetary Science Division (PSD) funding rates are high, the lunar community has been energized by the aggressive timeline of the Artemis program, and new companies are growing their deep space capabilities to support planetary exploration. In parallel, HEOMD has seen the benefits of leveraging commercial partners for human spaceflight through SpaceX's successful crewed Demo 2 mission. These novel ways of engaging with the commercial space sector are shifting risk to outside of NASA, permitting the agency to take technical risks that would otherwise be untenable.

Cost and risk are thus inexorably linked. The PSD has started incorporating low-cost mission opportunities (including novel partnerships through the Commercial Lunar Payload Services_CLPS_-program) into its exploration portfolio, which we strongly support. However, none of the spacecraft in these programs have yet launched-or successfully landed; the public-private partnership experiment thus remains untested. While awaiting these missions, we should consider their impact to the planetary science community in either success or failure.

In this paper, we explicate the relationship between mission cost and risk, examine the state of low-cost missions in the Planetary Science Division, list the benefits of further commercial involvement to planetary exploration and the planetary science community, and provide a set of incentives that would evolve beyond the CLPS program by attracting further engagement by commercial space companies during the next decade.

\section{The Relationship Between Cost and Risk}

Risk is the combination of the probability of an undesirable event and its consequences. There are different types of consequences: technical, financial, schedule, safety, political, etc. Reliability is the probability that hardware will perform as designed within a set of constraints.

Cost and risk are inexorably linked. The more expensive a mission is, the more impactful the consequences of failure are, leading to a desire to minimize risk. Spacecraft and component reliability can be improved by rigorous project management and additional testing. However, those risk mitigation strategies are expensive and lengthen schedules, resulting in decreased mission cadences in the absence of additional funding. As a spacecraft program proceeds (and especially if cost or schedule overruns occur), it can drift into the self-reinforcing space spiral (Figure 1; Wertz et al. 2011). The result is a culture of risk aversion, high cost, and low mission rates.

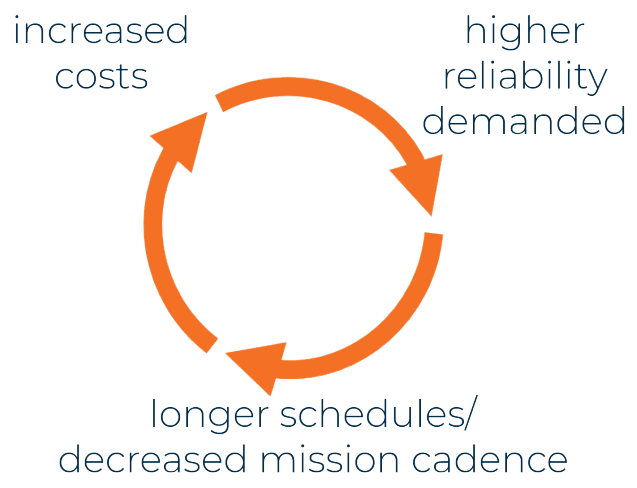

Figure 1. The "space spiral" (Wertz et al. 2011)

\section{The Original Low-Cost Program: Discovery}

Discovery program was created as a low-cost mission program in response to several cost and schedule overruns in the 1980s. NASA had found itself in the "space spiral" and did not launch a new planetary science mission for 11 years between 1978 and 1989. Unlike all previous planetary missions, which were led by NASA centers, the model for Discovery was to have competed proposals led by scientists. Although the first two missions, NEAR and Mars Pathfinder, were directed missions, all subsequent Discovery missions were completed and PIled. 
The initial Discovery cost cap was \$150M in FY 1992 USD (\$301M FY 2020), not including the launch vehicle and Phase E operations. The launch vehicle was limited to a low-cost, mediumsized Delta II rocket, whose small size constrained spacecraft mass. Mission development time was limited to 36 months with an expectation of a launch every 18 to 24 months. Notably, a key philosophy of the program was that NASA had to "be prepared to cancel any non-performing missions, in any Phase, from A to C/D" (Neufield 2018). Maintaining this balance of risk and speed with a consequence of cancellation was central to keeping costs low.

Discovery missions were originally intended to "proceed from definition to flight in less than three years, combining well-defined objectives, proven instruments and flight systems, strict cost limits and acceptance of a greater level of risk" (Savage 1992). But following four mission failures across the agency in 1999', NASA reinstated more rigorous systems management and oversight, extending development from 36 months to 45 then 51, allowing cost caps to be exceeded without cancelation, and selecting increasingly elaborate mission proposals (Neufield 2014).

Today, despite a stated cost-cap of \$500M, Discovery budgets regularly exceed that figure. Figure 2 shows the total cost of Discovery missions (except for Kepler, which was funded through the Astrophysics mission directorate) normalized to FY 2020 USD. ${ }^{2}$ (Note that Lucy and Psyche are still in development, so their costs may change.) These data show that even when correcting for inflation, the absolute cost of Discovery missions has grown with time. The cost growth is driven largely by strategic NASA decisions including (1) the removal of the launch vehicle cost from under the cost cap, (2) the removal of Phase E costs from under the cost cap, (3) the addition of the possible use of radioisotope power systems, and (4) descent into the space spiral. Discovery missions can no longer be called low-cost.

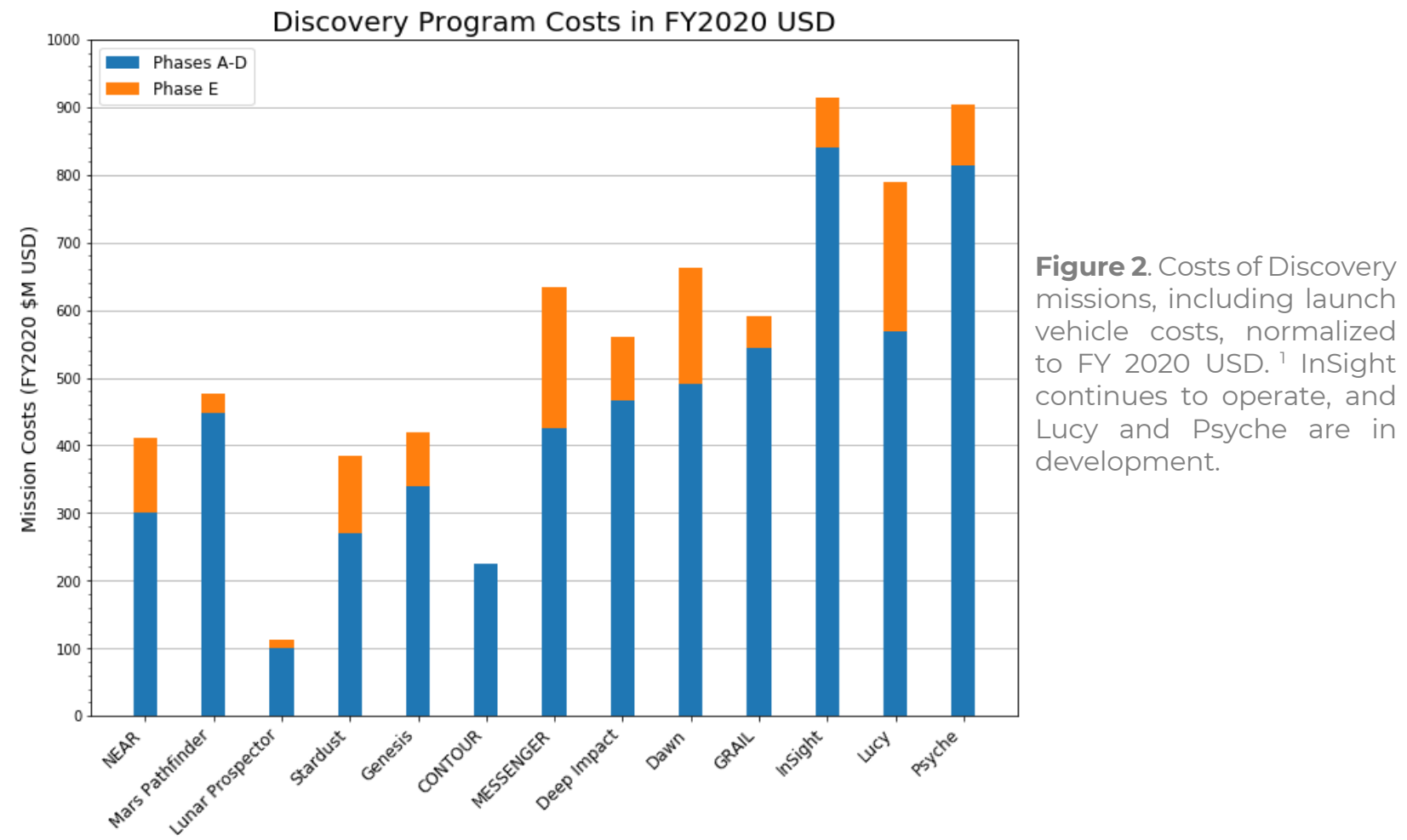

${ }^{1}$ Mars Polar Lander, Deep Space 2, Wide-Field Infrared Explorer, Clark Earth Observing Satellite

2 Data comes from the Planetary Society's Planetary Exploration Budget Dataset. Kepler is not included, The 2019 New Start Inflation Index for 2020 was used to convert real-year fiscal dollars to FY202O. Note that NASA switched to the full-cost accounting method in 2004, which accounts for some of the apparent cost growth. 
While these decisions have had positive impacts (e.g. allowing a wider diversity of targets achievable within the program), the cost growth disincentivizes NASA from the risk-taking and experimentation that typified the early Discovery era. It is no longer tenable to cancel overbudget or underperforming missions, as occurred with the Earth-orbiting satellite Clark, terminated in 1998 due to cost overruns and launch vehicle delays. The Discovery program is no longer structured to incentivize teams to take technical risks and keep costs down. Therefore, innovation and/or use of new technology is implicitly suppressed.

\section{The New Low-Cost Programs: SIMPLEx and CLPS}

To be clear, 2020 NASA is markedly different from the NASA that did not launch a deep space mission for 11 years in the 1980s. Today, the PSD has made improvements towards a better-balanced mission portfolio in terms of risk and cost, the decadal survey process provides a clear roadmap for decision-making, and NASA is experimenting with low-cost mission programs such as SIMPLEx and CLPS. The SIMPLEx cost cap is \$55M excluding launch, and the first round of contracts for lunar payload delivery were $\$ 77.3 \mathrm{M}$ to $\$ 97 \mathrm{M}$ including launch. These programs provide a mechanism for the technical risk-taking that defined the early Discovery missions.

Nearly 30 years after the first Discovery mission was selected in 1992, we find ourselves at a technical crossroads not unlike that which partly propelled the early Discovery missions: increasingly capable technology in smaller packages. The CubeSat platform, created in 1999 as a university teaching tool, hit a milestone in November 2018, when NASA's twin MarCO CubeSats successfully relayed InSight's entry, descent, and landing data from Mars to Earth. MarCO, which leveraged commercial off-the-shelf technology (COTS) developed for the lowEarth orbit satellite market, demonstrated that CubeSats in particular and SmallSats (<180 kg dry mass) more broadly can function in deep space.

Recognizing the promise of SmallSats for planetary exploration, NASA created the SIMPLEx program, first solicited in 2014; the last selection round occurred in 2019. Rated as Category 3 with Class D payloads, SIMPLEx missions inherently allow a "medium" amount of technical risk. (Categories and Classes are defined by NASA Procedural Requirements 7120.5E and 8705.4, respectively.) SIMPLEx allows for non-traditional spacecraft providers including universities, small businesses, and other organization that do not have the sources to execute a Discoveryclass or greater mission. At the time of writing, no SIMPLEx missions have launched, so the most challenging test for these missions remains.

In parallel, over the past decade, NASA has supported the growth of the commercial space industry through programs such as Commercial Cargo, Commercial Crew, and now CLPS, which will send NASA payloads to the lunar surface on commercial landers. The key difference between these programs and those with traditional prime contractors is that the contracts are fixed-price with payment upon meeting milestones. Programmatic risk is mitigated through the selection of multiple companies at the earliest project phases. Through competition and contract structure, these public-private partnerships incentivize providers to minimize project cost growth through innovative technical solutions, as demonstrated by SpaceX's reusable rocket boosters.

\section{Why More PSD Commercial Opportunities Will Benefit Planetary Science}

By expanding opportunities for the commercial sector to support planetary exploration, we predict the following downstream benefits to the planetary science community and more broadly to solar system exploration:

1. Sharing of Risk. For CLPS, the shift of risk from NASA to CLPS providers allows NASA to support technical risk from which it will benefit without bearing the full load of 
programmatic consequences should a failure occur. For SIMPLEx, the lower cost of these missions and their limited number of science goals means that failure will not have significant consequences compared to a more expensive and strategically important Flagship mission, for example.

2. Rapid Technology Iteration. Deep space flight opportunities are currently very limited, leading to a reliance on heritage technology. An increase in flight opportunities for novel technology and the relatively low consequences of failure for low-cost missions will facilitate a faster rate of technical development. This has been clearly demonstrated in LEO, typified by Earth observation companies such as Planet.

3. Opportunities to Visit Unexplored or Underexplored Targets. While there are no clear quantitative metrics to compare missions' science return (see Dillon and Madsen, 2015, for one attempt to use publication rates), there is arguably inherent value in having low-cost missions sent to a diverse set of solar system bodies-even if these missions produce data of limited quality/quantity_because they reduce risk for future, more capable missions.

4. Availability of New Mission Architectures. Smallsats' small size permits new mission architectures such as constellations composed of multiple CubeSats. What new science questions can we tackle with an increase in temporal and spatial coverage? Notably, having multiple spacecraft is itself a form of risk reduction should one spacecraft fail.

5. Mission Workforce Training and Diversification. An increase in flight opportunities is also an increase in leadership opportunities for future Discovery, New Frontiers, and Flagship mission PIs and Project Scientists. CLPS and SIMPLEx can serve as a lower-stakes training ground and wider pipeline to capture the best talent across the planetary science community, including historically underrepresented demographics.

6. Broadening of Planetary Science Career Opportunities. NASA PSD has built a strong community based upon funding of research projects and $\mathrm{PI}$-led mission opportunities that have led to the education of an increasing number of the next generation. However, few opportunities exist for planetary scientists to use their terminal degrees in the commercial space sector; this paper's first author is a rare exception. Funding that supports commercial space companies, particularly the new entrants within the past decade or two, will lead to further collaborations between the planetary science community and commercial space companies (which are largely composed of engineers). As the two communities become further enmeshed, eventually a planetary scientist job pipeline could be established from academia to the commercial space sector, supporting community employment goals (Frank et al. 2020).

\section{How NASA Can Attract Commercial Sector Involvement}

By creating opportunities that enhance participation by the full spectrum of commercial sector organizations, NASA could increasingly benefit from their growing capabilities. Some examples are given below.

\section{Have sensitivity to and flexibility in partnership \& contracting}

The structure of an opportunity-be it CLPS, SIMPLEx, or a new program-will impact which organizations decide to apply to it. The term "commercial space company" or ones like it are often used without the recognition that there are many types of companies, including but not limited to prime contractors, venture-backed firms, and employee-owned entities. Companies, by their very existence, are motivated to produce profit for their shareholders, but their particular governance and funding situation will impact how appealing a particular NASA solicitation is.

As a specific example, the first author of this paper has worked at both a venture-backed start-up (Planetary Resources, the asteroid mining company) and First Mode (a bootstrapped, employee-owned space and mining consultancy). Partnerships that require a company to put "skin in the game" (e.g. some \% of cash into the effort) are more likely to attract a venture- 
backed company with relatively high cash on hand (but not necessarily a revenue stream) compared to a bootstrapped company that is profitable but has neither the cash available or risk tolerance to participate in a partnership. For the latter, a fixed-price contract is a more desirable funding vehicle.

\section{Create sustainable, reliable funding opportunities}

Many of the CLPS providers are relatively small and young. For these small companies, it takes an enormous amount of effort and capital to build the team, infrastructure, and facilities required to design and develop a spacecraft. If a company successfully lands their spacecraft with NASA payloads on the Moon, but they do not have a follow-on contract with NASA, their only solution may be to lay staff off.

Thus, if NASA wants to capitalize on the success of the CLPS program, sustaining these companies through follow-on funding opportunities should be considered as they develop a broader customer base. Following successful missions, create clear pathways for CLPS providers to support Discovery, New Frontiers, and Flagship missions, by providing subsystems, flight demonstrations, or secondary payloads. This will ensure that the institutional knowledge in low-cost planetary exploration developed in these companies continues to benefit NASA, planetary scientists, and the companies themselves.

\section{Accepting more risk}

The existence of SIMPLEx and CLPS (as well as other more risk-tolerant programs across SMD) demonstrates NASA's increasing willingness to accept more technical risk on its projects. However, because none of these missions have launched, NASA's tolerance of failure in these programs is thus far untested. How will NASA react if the first CLPS mission fails like SpacelL's Beresheet Lunar Lander failed in 2019? What if the second mission fails as well? The third? What is an acceptable failure rate? How much does the root cause of the failure matter?

There are no easy answers, and we should steel ourselves for the prospect of failure and consider the most constructive ways to respond. NASA's response to the four mission failures in 1999 was to lengthen schedules, increase budgets, and mitigate risk through additional systems management (Neufield 2018). Subsequent Discovery missions have been extremely successful in their science return and discoveries. However, it should be acknowledged that an implicit trade exists between the money spent per mission and the number of missions that are possible within a finite budget (Figure 2). What scientific discoveries have not been made because funds were spent on unnecessary risk mitigation strategies for fear of failure?

If a CLPS or SIMPLEx mission fails, NASA should carefully examine the failure for its root cause and change course as needed rather than cancel the program. Striking the right balance is hard, but for these low-cost missions, risk reduction need not be the only reaction to failure. SpaceX, which has created highlight reels of its most spectacular rocket failures, ${ }^{3}$ demonstrates that an organization can experience failure without detriment to its public image. Arguably, it is this willingness to embrace failure and rapidly iterate using lessons learned that led SpaceX to successfully complete the first commercial water landing of astronauts returning from orbit in its Dragon capsule on August 3, 2020-45 years since NASA's last Apollo water landing.

\section{Provide clear requirements before and during project execution}

The more specific NASA can be in its solicitations and overarching strategy, the easier it will be for commercial companies to respond to opportunities. For example, the Lunar Terrain Vehicle (LTV) and Lunar Surface Science Mobility Systems (LSSMS) RFIs released in 2020 requested information from organizations on their capabilities. However, neither RFI included

\footnotetext{
3 "How Not to Land an Orbital Rocket Booster" SpaceX. <https://www.youtube.com/watch?v=bvim4rsNHkQ>
} 
specific landing sites with coordinates, nor a list of targets, which made it difficult to respond to. This is a classic chicken-and-egg problem: NASA is asking companies what they are capable of, and companies need to know what NASA specifically needs so they can adequately respond.

During a project, wherever there is an interface between two organizations, there is ample opportunity for miscommunication, particularly when the cultures of the organizations are different. NASA already has a hard time defining high-risk mission requirements internally: Bitten et al. (2013) showed that teams on Category 3, Class C/D missions struggle to shed the habits of a rigorous systems management, particularly when it comes to reviews. Anecdotally, this seems to still be the case in 2020. One solution is to create a task force that identifies and closes gaps in guidance for Category 3, Class C/D missions and outlines strategies for streamlining them. This task force could also critically evaluate lessons learned from the Faster, Better, Cheaper era missions, particularly the first 10 missions that had a $90 \%$ success rate (McCurdy 2003).

Implementing these methods and lessons in SIMPLEx, CLPS, and future programs will help prevent them from entering the "space spiral" (Figure 1) and ensure that science return is maximized for NASA's investment in these exciting missions.

\section{Conclusion}

\section{"The largest obstacle to low-cost innovation is the belief that it cannot be done."} (McCurdy 2003)

\section{References}

Bitten, R. et al. (2013) Assessing the Benefits of NASA Category 3, Low Cost Class C/D Missions. Proceedings from the 2013 IEEE Aerospace Conference.

Dillon, R.L. and P.M. Madsen (2015) Faster-Better-Cheaper Projects: Too Much Risk or Overreaction to Perceived Failure? IEEE Transactions on Engineering Management 62, 141-149.

Frank, E.A., et al. (2020) Normalizing non-academic career paths in planetary science. White paper submitted to the 2023-2033 Planetary Science Decadal Survey.

Launius, R. and H.E. McCurdy, Eds. (2016) Seeds of Discovery: Chapter in the Economic History of Innovation within NASA.

McCurdy, H.E. (2003) Faster, Better, Cheaper: Low-Cost Innovation in the U.S. Space Program. The Johns Hopkins University Press. Baltimore, MD.

Neufeld, M.J. (2018) The Discovery Program: Competition, Innovation, and Risk in Planetary Exploration. In: Launius R., McCurdy H. (eds) NASA Spaceflight. Palgrave Studies in the History of Science and Technology. Palgrave Macmillan, Cham.

Savage, D.L. (1992) NASA Announces Plans for Small Planetary Missions, NASA Press Release 92-05-8.

Wertz, J.R. et al. (2011) Methods of Achieving Dramatic Reductions in Space Mission Cost. Proceedings from the AAIA Reinventing Space Conference. Los Angeles. 